
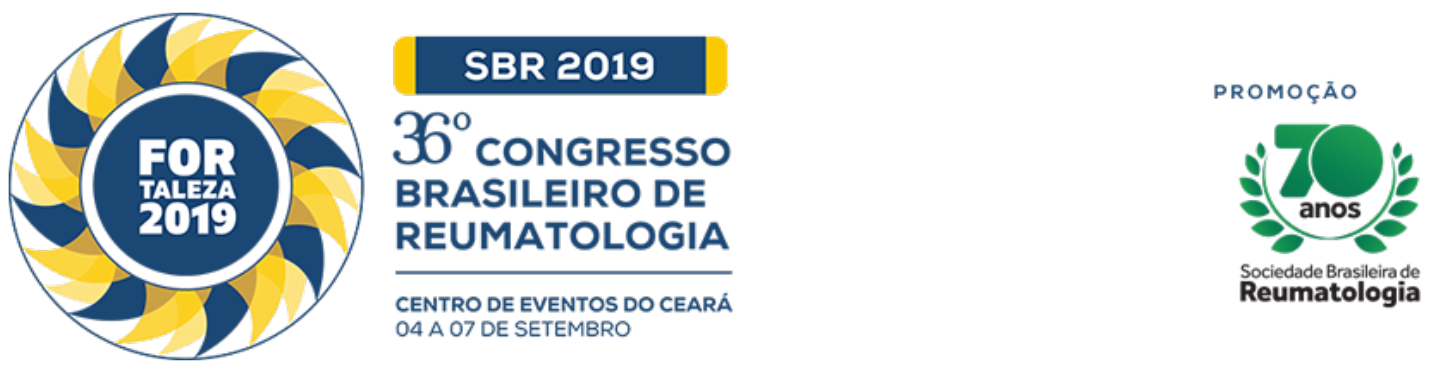

\title{
MTX-INDUCED PNEUMONITIS IN A PATIENT WITH RHEUMATOID ARTHRITIS
}

Vitalina de Souza Barbosa (Instittuto Cora Coralina, Goiânia, GO, Brasil), Heloísa Gouveia Machado (Universidade Federal de Goiás, Goiânia, GO, Brasil), Israel de Souza Barbosa Carneiro (Universidade Federal de Goiás, Goiânia, GO, Brasil), Pedro Paulo Teixeria e Silva Torres (Universidade Federal deGoiás, Goiânia, GO, Brasil), Rosane Gouveia Vilela Machado (Universidade Federal de Jataí, Jataí, GO, Brasil)

\section{BACKGROUND}

Drug-induced interstitial lung disease (ILD) is a rare but serious adverse event. Methotrexate-Induced ILD is the prototype of drug-induced lung toxicity in patients with rheumatoid arthritis (RA).

\section{CASE REPORT}

A 77-year-old female with a diagnosis of RA on $11 / 22 / 18$, started prednisolone $10 \mathrm{mg} / \mathrm{d}$ and MTX $10 \mathrm{mg} / \mathrm{w}$ with an increase in dose to $15 \mathrm{mg} / \mathrm{w}$ on $12 / 07 / 18$. She underwent chest CT on 02/05/18 which showed fibroatelectasic densities in the upper left lobe associated with diffusely distributed calcified nodules and micronodules of residual aspect (medical history of tuberculosis). On 03/05/19 the patient was admitted to the hospital with progressive dyspnea and dry cough that had started 10 days priorly. She denied expectoration or fever. A new chest CT (03/05/19) showed reticular pattern and patchy ground-glass opacities with diffuse and bilateral distribution, but no clear subpleural predominance, findings that were suggestive of interstitial lung disease. Chest CT (03/12/19) showed extensive diffuse bilateral patchy ground-glass opacities associated with inter and intralobular interstitial thickening that predominated in the upper and middle lobes. Honeycombing was absent. Transbronchial pulmonary biopsy was performed $(03 / 16 / 19)$ showing rare alveolar spaces with homogeneous-looking fibrosis and lymphoid aggregates, which did not allow for the diagnosis of usual interstitial pneumonitis due to scarcity of the material. Alveolar bronchial lavage culture was negative $(03 / 16 / 19)$. The patient was assumed to have Methotrexate-Induced ILD, and a 3 day pulse therapy with methylprednisolone $1 \mathrm{~g} / \mathrm{d}$ was administered. MTX was suspended and prednisone $30 \mathrm{mg} / \mathrm{d}$ was started. She was discharged from hospital on 03/23/1919 with significant improvement of the symptoms. Control chest CT (09/04/19) showed diffuse IDL with predominance of ground glass and reticular opacities, which might represent an initial nonspecific fibrosing component, but had improved in relation to the chest CT performed on 03/12/19.

\section{CONCLUSION}

Although rare, this is a well-documented case of MTX-induced pneumonitis. The diagnosis of MTX-induced pneumonitis is based on clinical evaluation, imaging and biopsy, as well as on the improvement with the use of corticosteroids and MTX suspension. The clinician should be alert for ILD-induced MTX in elderly patients with sudden onset of dyspnea. 